\title{
Influence of renewable power fluctuations on the lifetime prediction of lithium-ion batteries in a microgrid environment
}

\author{
Adrian Soto, Alberto Berrueta, Pablo Sanchis, Alfredo Ursúa \\ Department of Electrical, Electronic and Communication Engineering \\ Institute of Smart Cities \\ Public University of Navarre (UPNA) \\ Pamplona, Spain \\ adrian.soto@unavarra.es
}

\begin{abstract}
This contribution analyses lifetime estimation errors due to the effect of power fluctuations in lithium-ion batteries connected to microgrids when different time steps are used for the calculations. Usually, not every second data are available or the computational cost is excessively high. Those facts result in the use of larger time steps. However, the increase of the time steps may turn out in too optimistic predictions. Data from a real microgrid make it possible to optimize calculation times while keeping low errors. The results show that when 1 minute time step is set, the computation time is reduced by 14.4 times while the lifetime overstatement is only 3.5-5.2\% higher, depending on the aging model.
\end{abstract}

Keywords- Battery, Electrical energy storage, Lithium ion cell, Micro-grid, Power fluctuations, Renewable energy.

\section{INTRODUCTION}

The modernizing of the electric grid is an unstoppable and necessary fact where the renewable energies are becoming indispensable. Due to the rapid cost reduction of Li-ion batteries, this technology is expected to strongly increase its use as storage units in grid-connected systems, particularly large plants and self-consumption installations. For instance, the energy storage systems (ESS) may be used to fulfil the grid codes imposed by the transmission system operators. Conversely, a different application consists of introducing an ESS in a microgrid to store the generation surplus and discharge the ESS when the load exceeds generation.

In order to analyze the profitability of a self-consumption system, the lifetime of the different components, and particularly the ESS, needs to be anticipated. This is an important issue, since the ESS may represent around $50 \%$ of the initial investment [1]. Generally, the economic analysis are performed by simulation. When these simulations are carried out, the electrical models of the components that conform the system are required. These models take different facts into account, such as the power generation resulting from the available renewable source, or the aging of the ESS. The complexity of the model needs to be increased in order to achieve high accuracy, leading to higher computational requirements.

This fact evokes that in the most of those applications, lifetime predictions are usually tackled with a $0.25-1 \mathrm{~h}$ time step [2], [3]. Several aging models have been published in the top rated research journals for the purpose of predicting the battery life. These models take into account a number of parameters, such as battery current, depth of discharge $(D O D)$, state of charge $(S O C)$ or temperature.

We would like to acknowledge the support of the Spanish State Research Agency (AEI) and FEDER--UE under grants DPI2016-80641-R and DPI2016-80642-R and of Government of Navarra through research projects PI020 RENEWABLE-STORAGE and 0011-1411-2018-000029 GERA.
During $0.25-1 \mathrm{~h}$ time step, the power generation is either the mean of the generated power or a synthetic composition from average irradiation data. Due to the long time step, the current ripple generated by the fluctuations of the power generation, might not be taken into consideration, what may result in an undue battery life prediction. If a larger time step is set, those fluctuations are filtrated, thus, some parameters of the aging model as $D O D$ or battery current are not duly analyzed.

In this contribution, we study the damage suffered by a Li-ion battery when operating under power fluctuations and we compare the aging of the cell when fluctuations are not taken into account, according to two of the most extended aging models. The aim of considering different time steps is to prove the importance of power fluctuations when estimating the lifetime of a Li-ion battery in a microgrid. The results obtained in this contribution show, on the one hand, the influence of these power fluctuations and, on the other hand, the reduction of the computational effort as the time step is increased. This relationship may help further researches to choose a correct time step in order to keep a low lifetime error while the computational effort is reduced.

The work is organized as follows: Section II presents the battery model applied for this contribution. This battery model is composed by two aging models, an equivalent circuit model (ECM) and a thermal model. Section III offers a brief description of the scenario of application. Finally, Section IV analyzes and compares the results obtained by both aging models and the computational effort.

\section{BATTERY MODEL}

Batteries are a collection of cells arranged in series and/or parallel. Cells are connected in series to provide a higher voltage, while parallel connections increase the capacity of the battery. In this contribution, a single cell is used to characterize the aging suffered because of the power fluctuations. As shown by C. Campestrini [4], the inhomogeneity between different cells of a typical battery is lower than $1 \%$. Based on this result, in this study we use a single cell and extrapolate the results to a battery pack.

The selection of the proper aging model is one of the most challenging issues when dealing with Li-ion batteries. Some models try to predict the aging of power applications, such as electric vehicles or hybrid vehicles. Adversely, other models are focused in stationary applications, such as self-consumption. Moreover, due to the wide variety of $\mathrm{Li}$-ion technologies available in the market, not every aging model is appropriate for all chemistries. As mentioned above, two aging models are used in this contribution a first one provided by the manufacturer and a second one for stationary applications proposed in 
the literature. These two models are described in the next subsection.

In order to develop both, the electrical and thermal model, a single cell has been characterized as explained in the next subsections. The selected cell is an NCR $18650 \mathrm{~B}$, manufactured by Panasonic. The nominal capacity $\left(C_{n}\right)$ of the cell is $3350 \mathrm{mAh}$, with a maximum discharge rate of $2 \mathrm{C}$. $C$-rate is defined as the relationship between the charge or discharge current and the nominal capacity, $C$-rate $=|i| / C_{n}$. The voltage range of the cell is $2.5-4.2 \mathrm{~V}$ [5].

The characterization tests were conducted in a climatic chamber having a controlled temperature of $25^{\circ} \mathrm{C}$. In addition, the cell temperature has been monitored by a NTC sensor attached to the center of the cell using foam tape. In such a way, the proper measurement of the surface temperature, avoiding the impact of the room temperature is ensured. For this purpose, it has been made use of the test bench showed in Fig. 1.

\section{A. Aging model}

In order to predict the end of life (EOL) of a battery, several aging models have been proposed in the last years. Commonly, aging models are divided in the literature in two main categories: post-processing models and performance-degradation models [6]. Post-processing models such as cycle counting lifetime model or Ah counting lifetime models, only offer information regarding the remaining useful lifetime. They usually depend on a single parameter, $D O D$ or $C$-rate, and the amount of cycle numbers.

Contrarily, performance-degradation models such as, electrochemical or ECM based aging models offer information respecting the remaining useful lifetime and the degradation of its parameters [6]. These models present greater accuracy, since they take into account several parameters $(D O D$, temperature, $C$-rate, etc). The computational cost of performancedegradation models is higher than the one of post-processing models. In addition, previously work is necessary to develop the performance-degradation models, as the data given by manufactures do not include information regarding performance-degradation models.

In this contribution we use two methods, the first one based on the information provided by the manufacturer and the second one on an advanced model. Two aging models are presented in order to study how the expected lifetime of a battery is affected by the disregard of the power fluctuations in models of different complexity and accuracy. The EOL is reached when the State of Health $(\mathrm{SOH})$, which is the ratio of

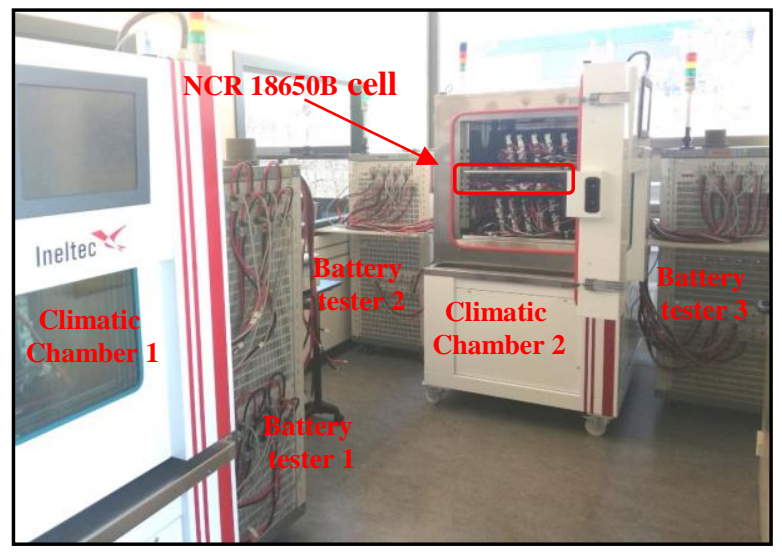

Fig. 1. Test bench used for cell characterization. the actual battery capacity to the initial capacity, decreases to $80 \%$.

\section{Model I: Manufacturer model}

One of the most extended ways of modelling battery aging is based on the number of equivalent charge-discharge full cycles $(E F C)$, as calculated in (1).

$$
E F C=\int \frac{|i|}{2 \cdot C_{n}} \cdot d t
$$

where $i$ is the cell current, $C_{n}$ the nominal capacity and $d t$ the time step.

This model is commonly used due to its low computational cost. The aging of the cell only shows a linear dependency on the charge throughput. The main drawbacks of this model, is firstly, that it is only valid for a single temperature, and secondly, that variables such as battery current, $D O D$ and battery voltage are not taken into account when estimating the aging process [7]. However, this method is one of the most common, since battery datasheets usually provide the required information. Fig. 2 shows the aging curve provided by the manufacturer.

In order to estimate the lifetime using the $E F C$ aging model, the relationship between the number of cycles and the actual capacity is obtained from reconstructing the curve given in the datasheet of the 18650 cylindrical Li-ion cell [5]. The end of life is achieved when the number of calculated $E F C$ reaches the cycle number, where, the actual capacity is lower than $80 \%$ of the nominal one. In this case, such a capacity corresponds to $2680 \mathrm{mAh}$.

\section{Model II: Advanced aging model}

More complex aging models are based on the calculation of calendar and cycle aging. Calendar aging is suffered by the battery during the storage time. Meanwhile, cycle aging occurs when the battery is being charged or discharged. These two aging phenomena are independent of each other. Therefore, they can be calculated separately and subsequently added [2], [3], [6]. A linear dependency of the capacity fade $\left(\Delta C / C_{n}\right)$ and time is set in calendar aging, while a linear dependency on the EFC is assumed when cycling aging is studied, as detailed in (2).

$$
\frac{\Delta C(d t, E F C)}{C_{n}(d t)}=-\left(\alpha_{c} \cdot d t+\beta_{C} \cdot \int \frac{|i|}{2 \cdot C_{n}} \cdot d t\right)
$$

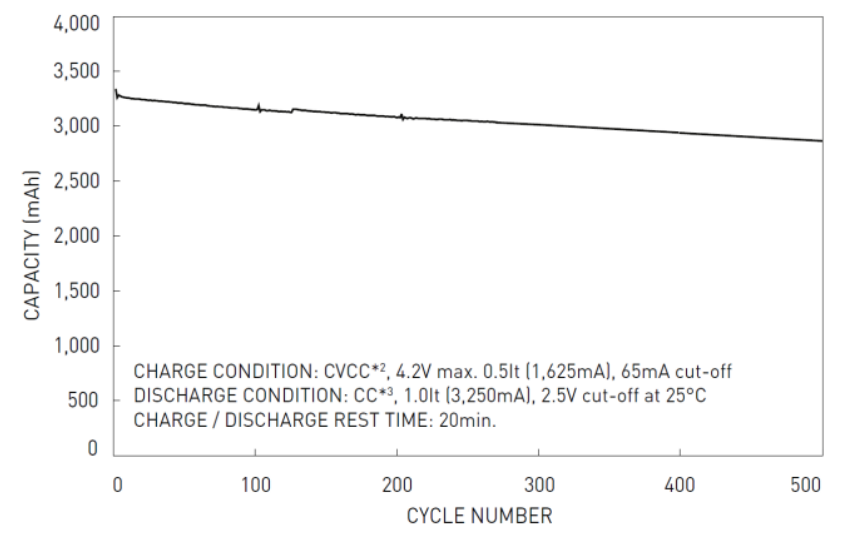

Fig. 2. EFC aging model delivered by the manufacturer [5]. 
where $\alpha_{C}$ represents the calendar aging and $\beta_{C}$ is related with the cycle aging. These parameters are not constant.

High values of $S O C$ and temperature have a negative effect on calendar aging as detailed in [8]. Cycle aging depends on current, average voltage and $D O D$ as reported in [9]. When high $C$-rates or $D O D$ are reached, the lifetime is decreased due to the effect of cycle aging. Both parameters $\left(\alpha_{C}\right.$ and $\left.\beta_{C}\right)$ can be calculated by means of (3) and (4). In these expressions, $v_{\text {cyc }}$ refers to the mean voltage on a cycle while $v_{\text {cell }}$ is the voltage of the cell and $T_{\text {cell }}$ is temperature. The values of the parameters are shown in Table I. In this method, the EOL is achieved when the capacity fade reaches a value of $20 \%$.

$$
\begin{gathered}
\beta_{C}=\left(b_{D O D} \cdot D O D+b_{0}\right)+b_{0}\left(v_{c y c}-b_{v 0}\right)^{2} \\
+b_{I} \cdot \exp \left(b_{\text {exp }} \cdot \frac{|i| \mid}{c_{n}}\right)
\end{gathered}
$$

TABLE I. BATTERY AGING MODEL PARAMETERS [2].

\begin{tabular}{cccc}
\hline & Parameter & Unit & $\Delta C$ \\
\hline \multirow{3}{*}{ Calendar } & $a_{v}$ & - & $2.716 \cdot 10^{5}$ \\
& $a_{0}$ & $V$ & 3.1482 \\
$a_{T}$ & $K$ & 6976 \\
& & & \\
& $b_{0}$ & - & $2.71 \cdot 10^{-5}$ \\
& $b_{v}$ & $V^{-1}$ & $3.14 \cdot 10^{-4}$ \\
& $b_{v 0}$ & $V$ & 3.683 \\
& $b_{D O D}$ & - & $1.61 \cdot 10^{-6}$ \\
& $b_{I}$ & - & $1.56 \cdot 10^{-5}$ \\
& $b_{\text {exp }}$ & $h$ & 1.8 \\
\hline
\end{tabular}

\section{B. Electrical model}

The kinetic behavior of the cell is modelled by means of a semi-empirical ECM with a non-linear dependence of the open circuit voltage $v_{O C}$ and the internal resistance $R_{D C}$ on the SOC [2].
$R_{D C}$ encompasses different phenomena that occur when the cell is operating. The phenomena can be described as: fast dynamic processes (ohmic phenomena and charge transfer trough the solid-electrolyte interface) and the diffusion processes (membrane diffusion and electrode diffusion). $R_{D C}$ follows Arrehenius law, however, its dependence with temperature is usually simplified to be linear. In such way, the computation is faster [10]. Therefore, $R_{D C}$ at a certain $S O C$ can be expressed as follows:

$$
R_{D C}=R_{0}+R_{1} \cdot T_{\text {cell }}
$$

Voltage drop and heat losses caused by the cell current are then included in the model. Heat losses, can be easily modelled according to Joule's law, $Q=i^{2} \cdot R_{D C}$. The electrical model is represented in Fig. 3 (a).

In this work $v_{O C}$ is obtained by means of a galvanostatic intermittent titration technique (GITT). This technique consist of a constant current (CC) charge or discharge state, followed by a rest time. This rest period ensures the thermal stability and reduces the effect of the $R_{D C}$ in the voltage measurement. In this work, a $\mathrm{C} / 2 \mathrm{CC}$ phase is done every $\triangle S O C$ of $5 \%$ followed by a rest of three hours. The relationship $S O C / v_{O C}$ is fitted by a tenth degree polynomial as shown in Fig. 3 (b).

The $R_{D C}$ values are also calculated every $\triangle S O C$ of $5 \%$. For this purpose, a current step $(\Delta I)$ of $10 \mathrm{~s}$ is applied, preceded by a rest period. From the obtained voltage drop $(\Delta V), R_{D C}$ can be easily obtained according to Ohm's law, $R_{D C}=(\Delta V) /(\Delta I)$. This is a common technique used in the literature by several authors [11]-[13]. In this contribution a current pulse of $\mathrm{C} / 2$ is defined, the results are shown in Fig. 3 (c).

\section{Thermal model}

Temperature is one of the most critical aspects of Li-ion batteries. Manufactures usually recommend operating the batteries between $15-35^{\circ} \mathrm{C}$ ambient temperature. If the temperature goes beyond this range, it evokes in a drastic lifetime reduction. Furthermore, if the cell temperature keeps growing it can result in a risky situation.

In order to predict the thermal behavior of a Li-ion cell, a thermal model is proposed in this work, and it is experimentally validated. The inputs of the model are the current of the cell $(i)$ and the room temperature $\left(T_{\text {room }}\right)$. The main reason of using these variables is that they can be easily measured. (a)

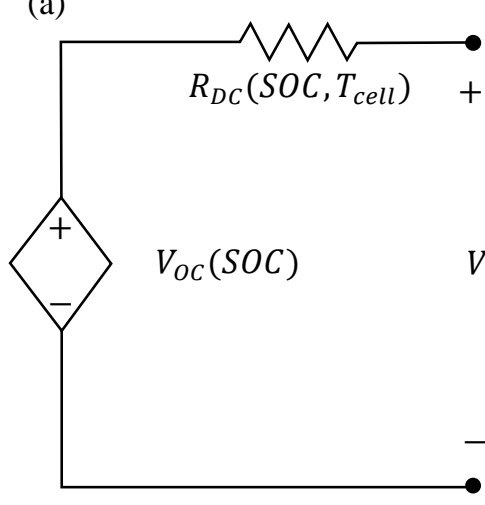

(b)

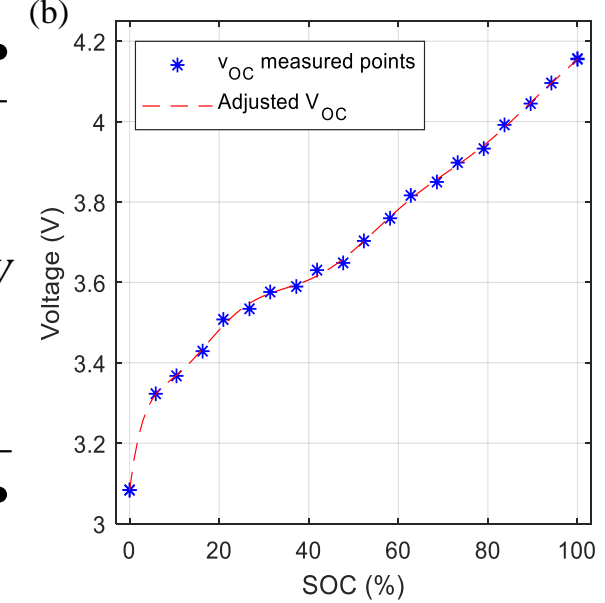

(c)

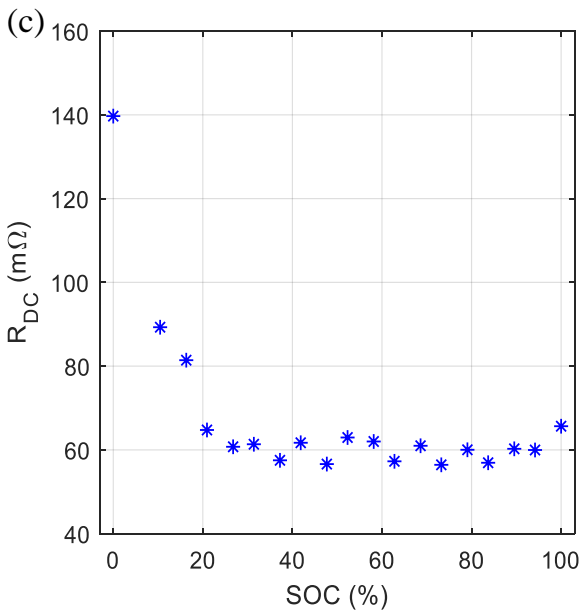

Fig. 3. Cell electrical model (a) Equivalent circuit, (b) $v_{O d}(S O C)$ relationship, (c) $R_{D d} /(S O C)$ relationship. 
The model consists of a heat generation source $\left(i^{2} \cdot R_{D C}\right)$, the thermal capacity of the cell $\left(C_{t h}\right)$ and a thermal resistance $\left(R_{t h}\right)$ which models the conduction, convection and radiation processes on the surface of the cell, as it is shown in Fig. 4. If the model is analyzed in steady state, the equation that governs the system is:

$$
T_{\text {cell }}-T_{\text {room }}=i^{2} \cdot R_{D C}\left(T_{\text {cell }}\right) \cdot R_{t h}
$$

Substituting (5) in (6) the cell temperature can be calculated as follows:

$$
\begin{gathered}
T_{\text {cell }}-T_{\text {room }}=i^{2} \cdot\left(R_{0}+R_{1} \cdot T_{\text {cell }}\right) \cdot R_{\text {th }} \\
T_{\text {cell }}=\frac{i^{2} \cdot R_{0} \cdot R_{t h}+T_{\text {room }}}{1-i^{2} \cdot R_{1} \cdot R_{\text {th }}}
\end{gathered}
$$

Temperature tests were conducted in a climatic chamber at a constant room temperature of $25^{\circ} \mathrm{C}$. The measurements of temperature were conducted on the steady state, for that purpose continuous charge and discharge cycles at $\mathrm{CC}$ were performed until the temperature of the cell was stable. For the purpose of keeping the temperature in the steady state, SOC variations were carried in the $20-95 \%$ range. As it can be observed in Fig. 3 (c), there exists a minimal variation of $R_{D C}$ in that range. Hence, $R_{D C}$ can be assumed to be constant in this $S O C$ range.

Taking into account the linearity shown in (5) and fitting the obtained temperature measurements with (7) all the parameters of the steady state can be obtained, as $R_{D C}$ at $25^{\circ} \mathrm{C}$ is known. It should be noted that $R_{D C}$ has been assumed to be constant only to calculate the parameters of the steady state equations.

The performance of the model under dynamic conditions corresponds with the $R C$ equivalent circuit shown in Fig. 4. $C_{t h}$ can be calculated from the test at different $C$-rates explained above. The parameters obtained from the conducted tests and R-squared $\left(R^{2}\right)$ are shown in Table II.

\begin{tabular}{ccc} 
TABLE II. & BATTERY THERMAL MODEL PARAMETERS. \\
\hline Parameter & Unit & Value \\
\hline$R_{0}$ & $\mathrm{~m} \Omega$ & 391.198 \\
$R_{1}$ & $\mathrm{~m} \Omega / \mathrm{K}$ & -1.111 \\
$R_{\mathrm{th}}$ & $\mathrm{K} / \mathrm{W}$ & 1.621 \\
$C_{\mathrm{th}}$ & $\mathrm{K} / \mathrm{J}$ & 159.724 \\
$R^{2}$ & $\mathrm{p} . \mathrm{u}$. & 0.991
\end{tabular}

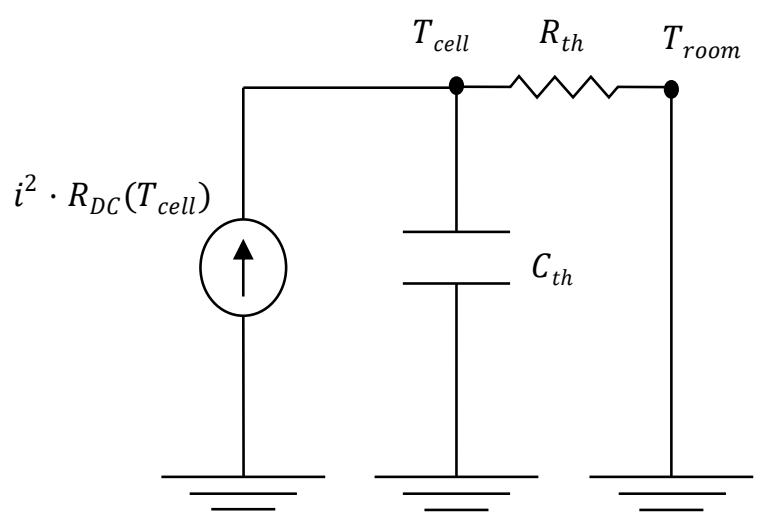

Fig. 4. Thermal model.

\section{SCENARIO OF OPERATION}

In order to generate a realistic scenario, the data for this work are taken from an experimental microgrid with renewable energy generation located on Navarre, Spain [14]. A diagram of the microgrid is shown in Fig. 5. The nominal power of the photovoltaic (PV) array is $4 \mathrm{kWp}$. The power consumption data is measured in a five-member family home located nearby the installation of the microgrid and emulated by means of an electronic load.

For this analysis, a period of one year is chosen when the microgrid operates normally. During this period, the energy is stored in case of excess of PV generation and is delivered when the consumption is higher than the PV generation as shown in Fig. 6.

The microgrid is composed by a commercial battery with the nominal capacity of $63 \mathrm{Ah}$, a nominal voltage of $51.8 \mathrm{~V}$ and a maximum $C$-rate of $0.9 \mathrm{C}$. The maximum and minimum cell voltage as defined by the manufacturer are $4.2 \mathrm{~V}$ and $3 \mathrm{~V}$ [15]. The battery is then, synthetically composed by 14 serial and 19 parallel NCR 18650 B cells, it should be taken into consideration that the battery is assembled with different cells; however, these cells were not available separately in the market. As explained, in this work the aging of a single cell is measured and extrapolated to a battery pack. A coulombic efficiency of $100 \%$ is assumed since, at ambient temperature, and low current rates, the efficiency of Li-ion batteries is almost unitary $\left(\eta_{C}>99.5 \%\right)[16]$. Nevertheless, the energy storage efficiency $\left(\eta_{E}\right)$ is modelled according to the quadratic relation which can be obtained from the electric model presented in Fig. 3 (a), being this efficiency dependent on the current and the SOC. Furthermore, a minimum (10\%) and maximum (95\%) SOC levels are fixed in order to enlarge the battery life.

\section{LIFETIME ESTIMATION}

Lifetime estimation is carried out in this section by means of both aging models presented in Section II. As it was explained, the models make it possible to predict the lifetime. Different time steps, from 1 second to 1 hour (3600 s) are used in order to show the influence of the time step in both models. In such a way, results of lifetime and computational effort are obtained for different time steps. For this work, every second real data measurements of the microgrid were available, if the

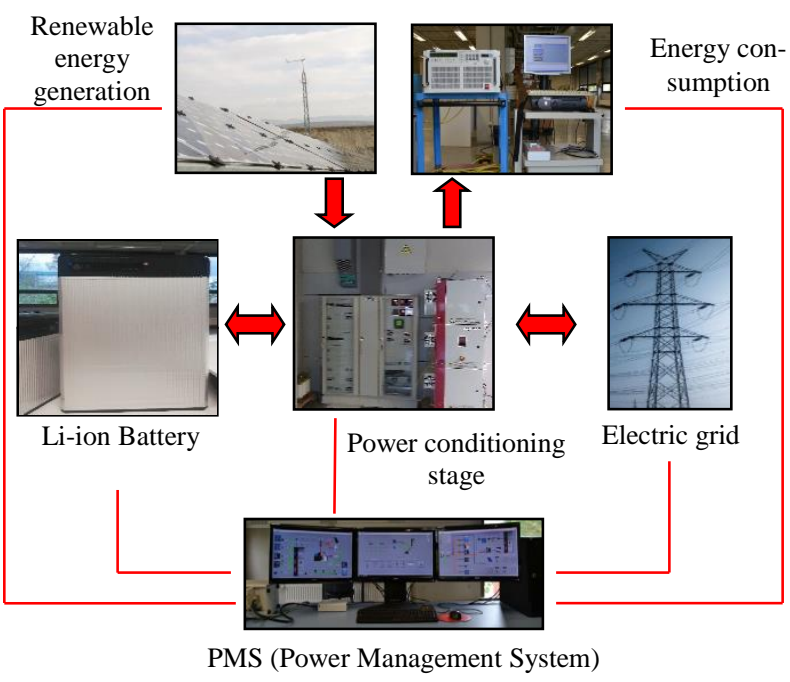

Fig. 5. Schematic diagram of the domestic microgrid. 


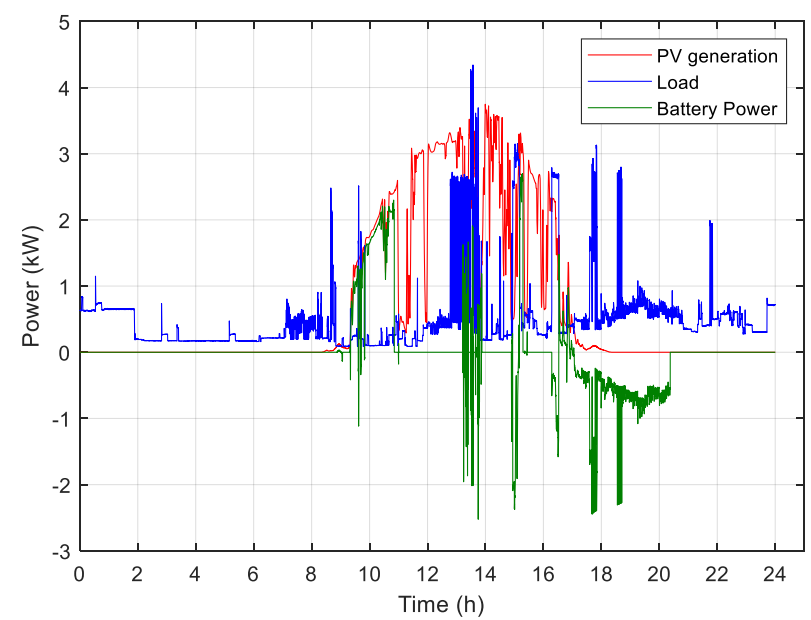

Fig. 6. Power flow of PV self-consumption on February 12.

time step is larger than a second, the current profile of the battery is assumed to be the average current during the time step.

As explained, the simulations were conducted for different time steps; the results show that there is a non-linear dependence on the predicted lifetime and time step. When the time step is increased also does the lifetime prediction augment. In opposition, when the step time is increased the computational effort is reduced. Therefore, battery life predictions may be too optimistic when using hourly average data.

If the manufacturer model is used, a difference of up to 0.55 years can be obtained in the lifetime estimation. Nonetheless, if a time step of 1 minute $(60 \mathrm{~s})$ is used, the lifetime prediction is only overestimate in a $3.5 \%$, whereas the time elapsed during the simulation is reduced by 14.4 times. Meanwhile, if the time step is increased up to $1 \mathrm{~h}(3600 \mathrm{~s})$, the error on the lifetime estimation raises to $14.4 \%$, while the elapsed time during the simulation is almost the same than that achieved when $1 \mathrm{~min}(60 \mathrm{~s})$ time step was used. The re-

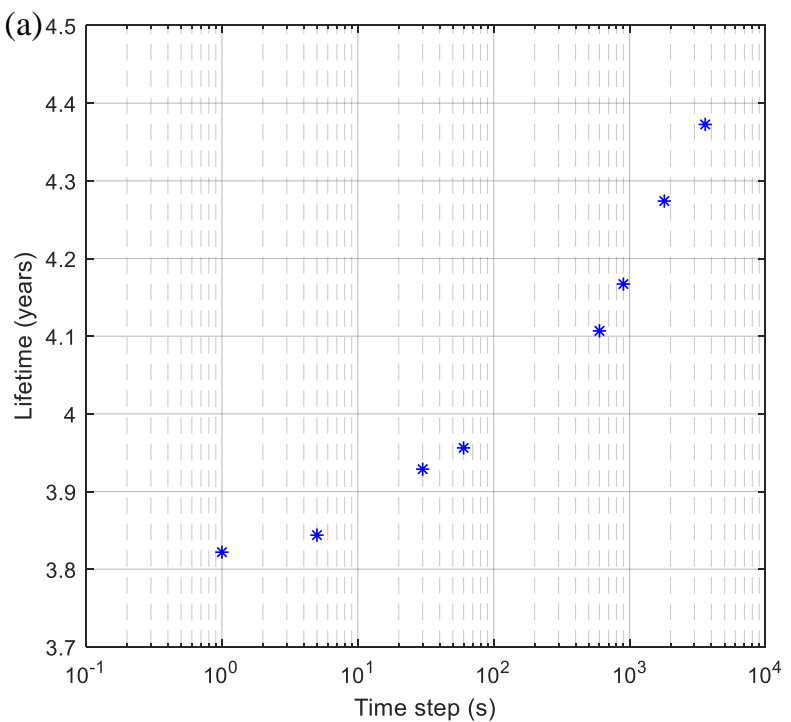

sults of the estimated lifetime for different time steps are presented in Fig. 7 (a), while the simulation times are shown in Fig. 7 (b).

If the advanced aging model is used, the discrepancy between the lifetime and the time step is even higher. Showing an error of 2.14 years when the lifetime is estimated using hourly time steps, instead of the real profile of one second time step, as shown in Fig. 8 (a). The fact of obtaining a greater difference than with the manufacture model is mainly due to the effect of different aging parameters, such as, temperature or $D O D$, that in the manufacturer model are not taken into account. When the step time of 1 minute $(60 \mathrm{~s})$ is analyzed, the difference in the computational effort becomes 16.5 times lower, while the deviation in the EOL is $5.2 \%$. There is no significant improvement in the time needed for the simulation if the time step is increased more than 1 minute, while the error due to the neglect of the power fluctuations keeps growing, as represented in Fig. 8 (b).

The manufacturer model predicts a lifetime around 4 years, while the advanced model predicts a lifetime around 10 years. This difference is caused because each model comes from a different source, as the advanced model is designed for stationary applications while the manufacturer model is a general model for mobility applications. The information provided by the datasheet only refers to cycle aging at a constant discharge of $1 \mathrm{C}$, in an application such the one studied in this work, usually, lower $C$-rates are managed. Thus, a larger lifetime should be reached in the EFC method. However, it is of significant importance that both models have shown a similar tendency.

\section{CONCLUSIONS}

When estimating the lifetime of a Li-ion battery in a microgrid, 10-20\% deviations can appear due to the time step of the simulation. Even for different aging models, from the simplest one to a more advanced model, the tendency is almost the same. If a larger time interval is used, the current ripples in the battery operation caused by the fluctuation of the renewable source are not taken into account and prediction can be too optimistic. The most accurate way is to predict the battery

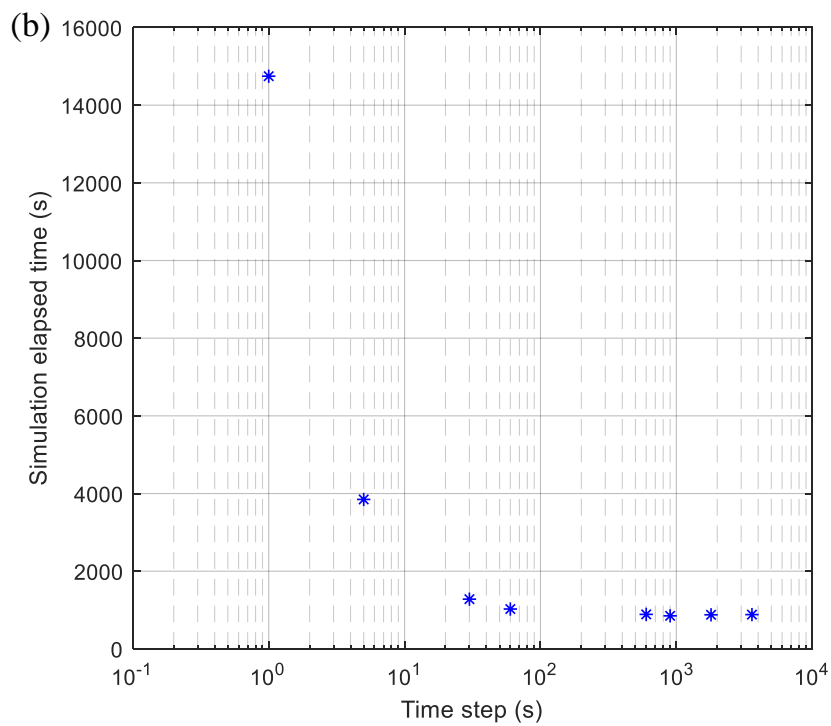

Fig. 7. Results of manufacturer aging model (a) Predicted battery life according to different time steps, (b) Time elapsed during the simulation according to different time steps. 

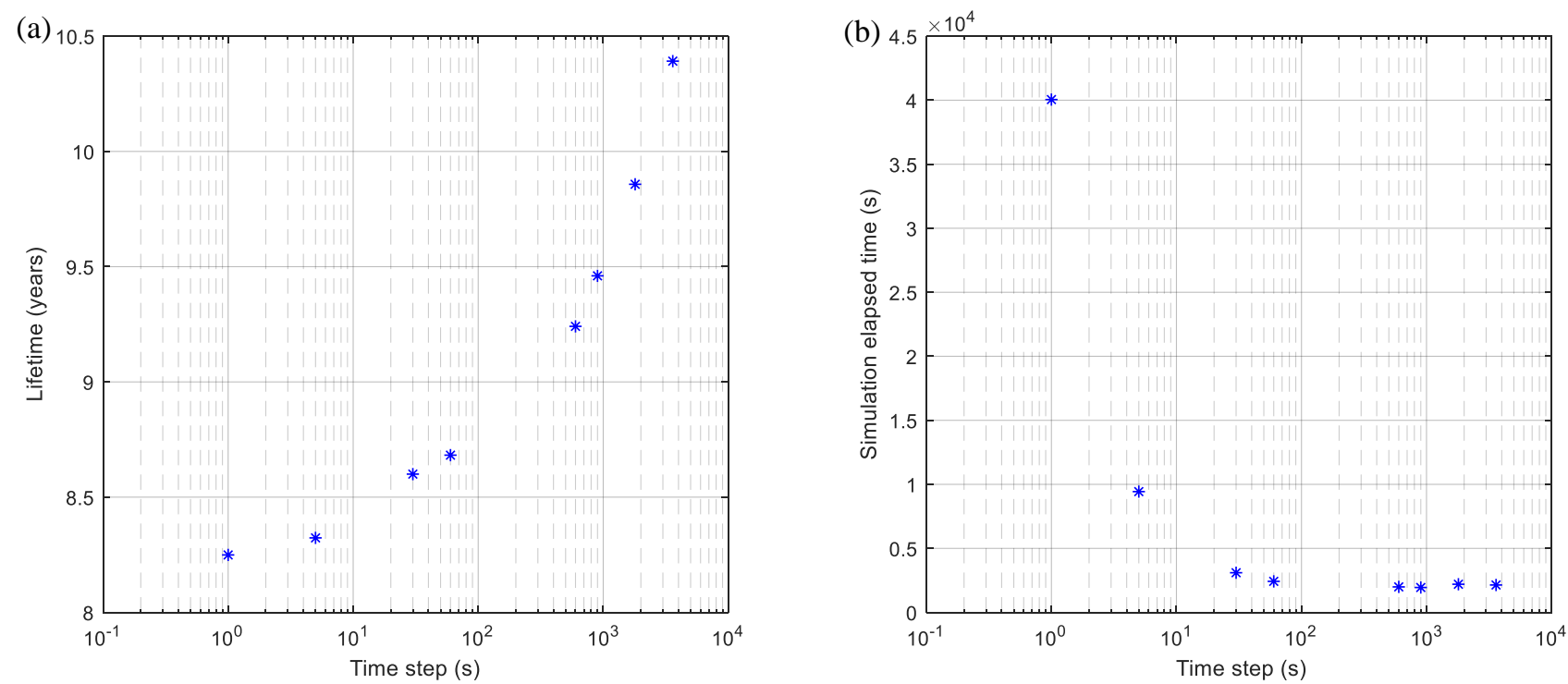

Fig. 8. Results of advanced aging model (a) Predicted battery life according to different time steps, (b) Time elapsed during the simulation according to different time steps.

aging using every-second data, however, this is not possible due to two main reason. Firstly, one second data are usually not available, and secondly, the computational cost can make it very difficult to obtain reliable results due to the complex equations that govern the aging models. In order to guarantee accuracy of the estimations at a reasonable computational cost, the analysis carried out in this paper makes it possible to choose the time interval while keeping the lifetime estimation error low. For instance, if the interval time of 1 minute is used, the lifetime error is only increased in a $3.5 \%$, while the computational cost is reduced by 14.4 times. The results show that the optimal relation between lifetime prediction accuracy and computational cost is achieved when a step time of 1 minute is chosen.

\section{REFERENCES}

D. Labrador and A. Kristen, "What's Driving the Cost of Residential Solar-Plus-Storage Systems?" [Online]. Available: https://www.rmi.org/whats-driving-cost-residential-solar-plusstorage-systems/. [Accessed: 12-Dec-2018].

[2] A. Berrueta, M. Heck, M. Jantsch, A. Ursúa, and P. Sanchis, "Combined dynamic programming and region-elimination technique algorithm for optimal sizing and management of lithium-ion batteries for photovoltaic plants," Applied Energy, vol. 228, no. February, pp. 1-11, 2018.

[3] I. San Martín, A. Berrueta, P. Sanchis, and A. Ursúa, "Methodology for sizing stand-alone hybrid systems: A case study of a traffic control system," Energy, vol. 153, pp. 870-881, 2018.

[4] C. Campestrini, P. Keil, S. F. Schuster, and A. Jossen, "Ageing of lithium-ion battery modules with dissipative balancing compared with single-cell ageing," Journal of Energy Storage, vol. 6, pp. 142-152, 2016.

[5] Panasonic, "Lithium ion Cell Type NCR18650B Specifications," $2018 . \quad$ [Online]. Available: https://www.elumeen.com/media/wysiwyg/produits/Elumeen_pa nasonic_NCR-18650B_en.pdf. [Accessed: 13-Mar-2019].

[6] D. I. Stroe, M. Swierczynski, A. I. Stan, R. Teodorescu, and S. J. Andreasen, "Accelerated lifetime testing methodology for lifetime estimation of lithium-ion batteries used in augmented wind power plants," IEEE Transactions on Industry Applications, vol. 50, no. 6, pp. 4006-4017, 2014.

[7] A. Berrueta, J. Pascual, I. S. Martin, P. Sanchis, and A. Ursua, "Influence of the Aging Model of Lithium-Ion Batteries on the
Management of PV Self-Consumption Systems," Proceedings 2018 IEEE International Conference on Environment and Electrical Engineering and 2018 IEEE Industrial and Commercial Power Systems Europe, EEEIC/I and CPS Europe 2018, no. 1, 2018.

[8] J. Schmalstieg, S. Käbitz, M. Ecker, and D. U. Sauer, "From accelerated aging tests to a lifetime prediction model: Analyzing lithium-ion batteries," 2013 World Electric Vehicle Symposium and Exhibition, EVS 2014, p. 6914753, 2014.

[9] J. Purewal, J. Wang, J. Graetz, S. Soukiazian, H. Tataria, and M. W. Verbrugge, "Degradation of lithium ion batteries employing graphite negatives and nickel-cobalt-manganese oxide + spinel manganese oxide positives: Part 2, chemical-mechanical degradation model," Journal of Power Sources, vol. 272, pp. $1154-1161,2014$

[10] A. Berrueta, A. Urtasun, A. Ursúa, and P. Sanchis, "A comprehensive model for lithium-ion batteries: From the physical principles to an electrical model," Energy, vol. 144, pp. 286-300, 2018

[11] M. J. Brand, M. H. Hofmann, S. S. Schuster, P. Keil, and A. Jossen, "The Influence of Current Ripples on the Lifetime of Lithium-Ion Batteries," IEEE Transactions on Vehicular Technology, vol. 67, no. 11 , pp. 10438-10445, 2018

[12] S. Li, S. Pischinger, C. He, L. Liang, and M. Stapelbroek, "A comparative study of model-based capacity estimation algorithms in dual estimation frameworks for lithium-ion batteries under an accelerated aging test," Applied Energy, vol. 212, no. December 2017, pp. 1522-1536, 2018

[13] A. Barai et al., "A comparison of methodologies for the noninvasive characterisation of commercial Li-ion cells," Progress in Energy and Combustion Science, vol. 72, pp. 1-31, 2019.

[14] J. Pascual, P. Sanchis, and L. Marroyo, "Implementation and control of a residential electrothermal microgrid based on renewable energies, a hybrid storage system and demand side management," Energies, vol. 7, no. 1, pp. 210-237, 2014.

[15] LG, "LGChem Catalog Global 2018." [Online]. Available: http://www.lgchem.com/upload/file/product/LGChem_Catalog_ Global 2018.pdf. [Accessed: 05-Dec-2018].

[16] A. J. Smith, J. C. Burns, S. Trussler, and J. R. Dahn, "Precision Measurements of the Coulombic Efficiency of Lithium-Ion Batteries and of Electrode Materials for Lithium-Ion Batteries," Journal of The Electrochemical Society, vol. 157, no. 2, p. A196, 2010 